\title{
Optimización de la terapia nutricional en el paciente crítico con COVID-19
}

\section{Nutritional therapy optimization in COVID-19 critically ill patients}

\author{
Francisco G. Yanowsky-Escatell' e Iván A. Osuna-Padilla*
}

${ }^{1}$ Hospital Civil de Guadalajara Dr. Juan I. Menchaca, Departamento de Medicina Interna, Servicio de Nutrición, Jalisco, México; ${ }^{2}$ Instituto Nacional de Enfermedades Respiratorias, Centro de Investigación en Enfermedades Infecciosas, Ciudad de México, México

Actualmente COVID-19, ocasionado por el nuevo coronavirus del síndrome respiratorio grave agudo 2 (SARS-CoV-2), representa una amenaza para la salud pública mundial. Los pacientes infectados tienen riesgo de desarrollar síndrome de dificultad respiratoria aguda; se ha reportado que 5 a $10 \%$ de la población infectada requerirá ingreso a una unidad de cuidados intensivos (UCl) y ventilación mecánica invasiva. ${ }^{1}$ Debido a la incapacidad del paciente en esa condición para alimentarse por vía oral, la terapia nutricional debe considerarse un componente del tratamiento integral de esta enfermedad emergente.

Una proporción importante de la población infectada de SARS-CoV-2 cursa con sobrepeso y obesidad; se ha reportado que un índice de masa corporal $>28 \mathrm{~kg} / \mathrm{m}^{2}$ constituye un factor de riesgo de severidad de la enfermedad (razón de momios $=5.872$, IC $95 \%=1.595-21.621$, $p=0.008) .{ }^{2}$ Sin embargo, la presencia de sobrepeso u obesidad no justifica la prescripción de ayuno en el paciente en estado crítico, ya que en otras condiciones clínicas se ha documentado que hasta $60 \%$ de los pacientes con obesidad que ingresan a la UCI presenta desnutrición, ${ }^{3}$ razón por la cual debe optimizarse la terapia nutricional con el fin de prevenir el deterioro o mejorar el estado nutricional. Independientemente del índice de masa corporal, la desnutrición en el paciente crítico es un grave problema al asociarse a mayor riesgo de infecciones, incidencia de úlceras por presión, incremento en los costos hospitalarios y mayor mortalidad. ${ }^{4,5}$
La enfermedad crítica evoluciona en dos fases: fase aguda (catabolismo) y posaguda (anabolismo). A su vez, la fase aguda se divide en dos periodos: el temprano (uno a dos días), caracterizado por inestabilidad metabólica e incremento severo en el catabolismo; y tardío (tres a siete días), definido por el desgaste muscular significativo y la estabilización de las alteraciones metabólicas. En la fase posaguda, el paciente puede mejorar, rehabilitarse o permanecer en estado catabólico/inflamatorio persistente y en hospitalización prolongada. ${ }^{6}$

El paciente crítico se encuentra en estado de estrés catabólico e inadecuada ingestión, lo cual lo predispone a desnutrición. ${ }^{7}$ El paciente crítico cuya estadía en la $\mathrm{UCl}$ es mayor de 48 horas debe ser considerado con riesgo de desnutrición, por lo que debe prescribirse terapia nutricional médica, definida por la European Society for Clinical Nutrition and Metabolism (ESPEN) como la provisión de nutrimentos por vía oral, enteral (nutrición enteral, NE) o parenteral (nutrición parenteral, NP). ${ }^{6}$

No existen datos concretos de las intervenciones nutricionales en los pacientes con COVID-19 y su impacto en el desenlace clínico. En la Tabla 1 se mencionan las recomendaciones de las guías para el manejo nutricional del paciente con COVID-19, emitidas recientemente por la British Dietetic Association ${ }^{8}$ y la ESPEN, ${ }^{9}$ en las que se incorporan sugerencias de la ESPEN, ${ }^{6}$ la American Society of Parenteral and Enteral Nutrition ${ }^{10}$ y la German Society for Nutritional
Correspondencia:

*Iván A. Osuna-Padilla

E-mail: ivan.osuna@cieni.org.mx

0016-3813/@ 2020 Academia Nacional de Medicina de México, A.C. Publicado por Permanyer. Este es un artículo open access bajo la licencia CC BY-NC-ND (http://creativecommons.org/licenses/by-nc-nd/4.0/).
Fecha de recepción: 09-04-2020

Fecha de aceptación: 29-05-2020

. 
Tabla 1. Recomendaciones para la terapia nutricional médica en pacientes críticos con COVID-19

\begin{tabular}{|c|c|}
\hline Proceso & Recomendación \\
\hline $\begin{array}{l}\text { Riesgo de } \\
\text { desnutrición }\end{array}$ & - Considerar con riesgo de desnutrición a todos los pacientes críticos con estancia mayor de 48 horas en la UCI. \\
\hline $\begin{array}{l}\text { Evaluación } \\
\text { nutricional }\end{array}$ & $\begin{array}{l}\text { - Evaluar peso y talla. En caso de no contar con básculas integradas a camillas, estimarlos con ecuaciones } \\
\text { predictivas. } \\
\text { - Valorar la estabilidad hemodinámica (dosis de vasopresores, tensión arterial media, equilibrio ácido-base, lactato } \\
\text { sérico). } \\
\text { - Cuantificar el aporte energético proveniente de medicamentos (uso de citrato, soluciones para diálisis, propofol, } \\
\text { infusiones de dextrosa). } \\
\text { - Evaluar el control metabólico a través de parámetros como glucosa y triglicéridos. } \\
\text { - Identificar a pacientes con mayor riesgo de desarrollar síndrome de realimentación (concentraciones de fósforo, } \\
\text { potasio y magnesio). }\end{array}$ \\
\hline $\begin{array}{l}\text { Terapia nutricional } \\
\text { médica }\end{array}$ & $\begin{array}{l}\text { - Establecer NE como primera vía de alimentación (primeras } 48 \text { horas). } \\
\text { - Utilizar sonda a estómago como primera opción o alimentación pospilórica, si existe intolerancia gástrica a pesar } \\
\text { del tratamiento con procinéticos o en presencia de alto riesgo de aspiración. } \\
\text { - Seleccionar fórmulas enterales poliméricas estándar ( } 1-1.5 \mathrm{kcal} / \mathrm{mL} \text { ) o densamente energéticas ( } 1.5-2 \mathrm{kcal} / \mathrm{mL} \text { ) } \\
\text { cuando se busca restricción de volumen. No existen beneficios con el uso de fórmulas especializadas para } \\
\text { enfermedades pulmonares. } \\
\text { - La ventilación mecánica invasiva en decúbito prono no es una contraindicación para la infusión de NE. } \\
\text { - Deberá implementarse NP en caso de contraindicación de NE, entre los tres y siete días de estancia hospitalaria. } \\
\text { Puede ser temprana y progresiva cuando exista contraindicación de NE en pacientes severamente desnutridos o si } \\
\text { existe intolerancia a la NE. } \\
\text { - Iniciar NP complementaria en los pacientes en quienes no se ha logrado alcanzar el } 100 \% \text { de los requerimientos } \\
\text { después de siete días de NE. } \\
\text { - Emplear formulaciones estandarizadas (tricámara) o individualizadas para la NP. } \\
\text { - Prescribir } 20-25 \mathrm{kcal} / \mathrm{kg} \text { de peso ideal; aportar } 70 \% \text { de los requerimientos los primeros tres días y de } 80 \text { a } 100 \% \\
\text { después del día } 3 . \\
\text { - Aportar } 1.3 \mathrm{~g} / \mathrm{kg} \text { de proteína (cantidad que deberá alcanzarse entre los días } 3 \text { y } 5 \text { ), incrementando el aporte en } \\
\text { 1.7-2.5 g/kg/día en el paciente con lesión renal aguda en tratamiento de reemplazo renal. }\end{array}$ \\
\hline $\begin{array}{l}\text { Monitoreo de la } \\
\text { terapia nutricional } \\
\text { médica }\end{array}$ & $\begin{array}{l}\text { - Verificar el volumen infundido de NE o NP. } \\
\text { - Vigilar la aparición de signos de intolerancia gastrointestinal (diarrea, distensión abdominal, vómito, presión } \\
\text { intraabdominal, residuo gástrico > } 500 \mathrm{~mL} \text { ) o de isquemia intestinal, balance hídrico y electrólitos séricos. } \\
\text { - Orientar la exploración física al estado nutricional para identificar posibles signos de deficiencias de nutrimentos. } \\
\text { - Monitorear la estabilidad hemodinámica y las dosis de vasopresores. Retrasar el inicio o progresión de la terapia } \\
\text { nutricional médica en pacientes con inestabilidad, dosis escalonadas o doble apoyo de vasopresor. }\end{array}$ \\
\hline $\begin{array}{l}\text { Periodo } \\
\text { posventilación } \\
\text { mecánica y } \\
\text { disfagia }\end{array}$ & $\begin{array}{l}\text { - Realizar prueba de deglución antes del inicio de la vía oral, una vez retirada la ventilación mecánica invasiva. } \\
\text { - Prescribir dieta modificada en consistencia en caso de alteración en la deglución. } \\
\text { - Continuar con NE en los pacientes cuya deglución no es segura. } \\
\text { - Prescribir NE vía sonda pospilórica si existe alto riesgo de aspiración; de no ser factible, utilizar NP durante el } \\
\text { periodo de rehabilitación de la disfagia. }\end{array}$ \\
\hline
\end{tabular}

Medicine ${ }^{11}$ para el paciente crítico, las cuales podrán modificarse de acuerdo con la evolución del conocimiento y de la epidemia.

La terapia nutricional médica dependerá de la condición clínica y grado de desnutrición del paciente. Se sugiere iniciar NE en las primeras 48 horas de estancia en la $\mathrm{UCl},{ }^{6}$ debido a sus beneficios en la mucosa gástrica, síntesis de hormonas y enzimas y regulación de la respuesta inmunológica. ${ }^{12}$ Si existiese contraindicación para NE, deberá instaurarse NP entre los tres y siete días. Se deberá considerar la utilización de NP temprana y progresiva en los sujetos severamente desnutridos, con contraindicación o intolerancia a NE. ${ }^{6,9}$
El aporte nutrimental debe incrementarse paulatinamente: en los primeros tres días se sugiere proporcionar $70 \%$ de los requerimientos energéticos y posteriormente incrementar hasta alcanzar 80 a $100 \%$. El aporte proteico debe ser de $1.3 \mathrm{~g} / \mathrm{kg} / \mathrm{día}$. Si durante la primera semana no es posible cubrir la totalidad de los requerimientos energéticos y proteicos del paciente mediante NE, deberá evaluarse de manera individualizada el inicio de NP. ${ }^{6,9}$

Aun cuando las guías internacionales sugieren la incorporación de protocolos de nutrición en las $\mathrm{UCl}$, diversos estudios han reportado que el conocimiento, entrenamiento y tiempo para la prescripción de la terapia nutricional médica son insuficientes en el equipo médico 
y de enfermería. ${ }^{13,14}$ Debido a lo anterior, la American Society of Parenteral and Enteral Nutrition sugiere considerar la nutrición como un proceso terapéutico en el manejo del paciente crítico e incorporar a un nutriólogo clínico,$^{15}$ profesional que se encarga de la prescripción, implementación y monitoreo de esta intervención. ${ }^{16}$

La pandemia de COVID-19 continúa propagándose en América Latina, lo cual incrementa la carga de los sistemas de salud públicos y privados. La atención de los pacientes con esta enfermedad no solo se concentra en las $\mathrm{UCl}$, también se lleva a cabo en otras áreas de hospitalización. Por lo tanto, conocer las implicaciones de la terapia nutricional médica y las recomendaciones nutricionales actuales optimizará el tratamiento y curso de COVID-19 en los pacientes.

\section{Bibliografía}

1. Poston JT, Patel BK, Davis AM. Management of critically ill adults with COVID-19. JAMA. 2020;323:1839-1841.

2. Jordan RE, Adab P, Cheng KK. COVID-19: risk factors for severe disease and death. BMJ. 2020;368:m1198. DOI: https://doi.org/10.1136/bmj.m1198

3. Robinson MK, Mogensen KM, Casey JD, McKane CK, Moromizato T, Rawn JD, et al. The relationship among obesity, nutritional status, and mortality in the critically ill. Crit Care Med. 2015;43:87-100.

4. Barker LA, Gout BS, Crowe TC. Hospital malnutrition: prevalence, identification and impact on patients and the healthcare system. Int J Environ Res Public Health. 2011;8:514-527.
5. Ruiz AJ, Buitrago G, Rodríguez N, Gómez G, Sulo S, Gómez C, et al. Clinical and economic outcomes associated with malnutrition in hospitalized patients. Clin Nutr. 2019;38:1310-1316.

6. Singer P, Blaser AR, Berger MM, Alhazzani W, Calder PH, Casaer MP, et al. ESPEN guideline on clinical nutrition in the intensive care unit. Clin Nutr. 2019;38:48-79.

7. Lee ZY, Heyland DK. Determination of nutrition risk and status in critically ill patients: what are our considerations? Nutr Clin Pract. 2019;34:96-111.

8. British Dietetic Association [Internet]. Bear D, Terblanch E. Birmingham. Critical Care Specialist Group Guidance on management of nutrition and dietetic services during the COVID-19 pandemic. 2020; Mar 23. Disponible en: https://www.bda.uk.com/resource/critical-care-dietetics-guidance-covid-19.html

9. Barazzoni R. ESPEN expert statements and practical guidance for nutritional management of individuals with SARS-CoV-2 infection. Clin Nutr. 2020;39:1631-1638.

10. McClave SA, Taylor BE, Martindale RG, Warren MM, Johnson DR, Braunsvhweig C, et al. Guidelines for the provision and assessment of nutrition support therapy in the adult critically ill patient: Society of Critical Care Medicine (SCCM) and American Society for Parenteral and Enteral Nutrition (A.S.P.E.N.). J Parenter Enteral Nutr. 2016;40:159-211.

11. Elke G, Hartl WH, Kreymann KG, et al. Clinical nutrition in critical care medicine - Guideline of the German Society for Nutritional Medicine (DGEM). Clin Nutr ESPEN. 2019;33:220-275.

12. Allen K, Hoffman L. Enteral nutrition in the mechanically ventilated patient. Nutr Clin Pract. 2019;34:540-557.

13. Mowe M, Bosaeus I, Rasmussen HH, Kondrup J, Unosson M, Rothenberg $E$, et al. Insufficient nutritional knowledge among health care workers? Clin Nutr. 2008;27:196-202.

14. Grammatikopoulou MG, Katsouda A, Lekka K, Tsantekidis K, Bouras E, Poulia KA, et al. Is continuing medical education sufficient? Assessing the clinical nutrition knowledge of medical doctors. Nutrition. 2019;57:69-73.

15. Tappenden KA, Quatrara B, Parkhurst ML, Malone AM, Fanjiang G, Ziegler TR. Critical role of nutrition in improving quality of care: an interdisciplinary call to action to address adult hospital malnutrition. J Parenter Enteral Nutr. 2013;37:482-497.

16. Terblanche $\mathrm{E}$. The role of dietitians in critical care. J Intensive Care S. 2019;20:255-257. 